\title{
Computational Modelling of Blood Flow Development and Its Characteristics in Magnetic Environment
}

\author{
Gopal Chandra Shit \\ Department of Mathematics, Jadavpur University, Kolkata 700032, India \\ Correspondence should be addressed to Gopal Chandra Shit; gopal_iitkgp@yahoo.co.in
}

Received 15 July 2013; Accepted 19 September 2013

Academic Editor: Aiguo Song

Copyright (C) 2013 Gopal Chandra Shit. This is an open access article distributed under the Creative Commons Attribution License, which permits unrestricted use, distribution, and reproduction in any medium, provided the original work is properly cited.

\begin{abstract}
Of concern in this paper is an investigation of the entrance length behind singularities in cardiovascular hemodynamics under magnetic environment. In order to get better interpretation of scan MRI images, the characteristics of blood flow and electromagnetic field within the circulatory system have to be furthermore investigated. A 3D numerical model has been developed as an example of blood flowing through a straight circular tube. The governing coupled nonlinear differential equations of magnetohydrodynamic (MHD) fluid flow are reduced to a nondimensional form, which are then characterized by four dimensionless parameters. With an aim to validate our numerical approach, the computational results are compared with those of the analytical solution available in the developed region far from the singularity. The hydraulic impedance by unit length within the developed flow region increases with the magnetic field. The time average entrance length with a greater precision on the unsteady case decreases with increasing magnetic field strength. The overall voltage characteristics do not depend on the developed flow field within the entry region.
\end{abstract}

\section{Introduction}

The steady and unsteady entrance flow region is important for biofluid dynamics, particularly the flow of blood in arteries with or without presence of magnetic field. It is important to understand and quantify the flow characteristics of blood in the entrance region such as near the origin of arterial branches, arterial constrictions, and aortic arch. It is also important for experimental models to create the appropriate inlet boundary conditions and for computational models to choose the length of the computational domain or mesh generation which requires the distance to be fully developed. Several investigators [1-4] have described the steady entrance flow and unsteady/oscillatory entrance flow $[5,6]$ in a circular tube. In spite of that, a huge number of studies on fully developed flow in a straight circular vessels are available in the scientific literatures. The analytical solution for steady flow first is described by Hagen-Poiseuille and unsteady flow by Womersley $[7,8]$. But no attempt is available to determine both the steady and unsteady entrance length using three-dimensional modelling of computational fluid dynamics approach in the presence of magnetic environment. Although $\mathrm{He}$ and $\mathrm{Ku}$ [9] have determined the unsteady entrance flow region in a straight tube by using spectral element simulation of the fully unsteady two-dimensional Navier-Stokes equations in the absence of magnetic field. For steady flow $\mathrm{He}$ and $\mathrm{Ku}$ [9] pointed out that the steady entrance length is proportional to the mean Reynolds number, wherein they established a relation of the form $L_{e} \approx 0.25 \mathrm{Re}$, in which $\operatorname{Re}=U_{0} a / \mathcal{\vartheta}, a$ being the tube radius, $U_{0}$ being the uniform entrance mean velocity, and $\vartheta$ being the kinematic viscosity. For pulsatile flow, the entrance length should vary sinusoidally with time.

Several studies are available toward the applications in magnetohydrodynamic blood flow of which most of them presented approximate solutions. There are no such attempts available for the development of three-dimensional modelling of MHD flow to obtain complete numerical solutions. An analytical approach is put forward by Gold [10], Vardanyan [11] and Abi-Abdallah et al. [12] for mathematical modelling of MHD flow in pipe with considering the effect of induced magnetic field under constant pressure gradient. These studies are restricted in a unidirectional fully developed region, whereas the other components of velocity and induced magnetic fields have been neglected. However, their analytical solutions are valid for any values of the Hartmann number (Ha). All these studies already made validation with 
the experimental as well as theoretical works of Hartmann [13], Hartmann and Lazarus [14], and Shercliff [1]. Later on Abi-Abdallah et al. [15] developed a mathematical model for MHD flow of blood to obtain velocity profiles and flow rate expressions by neglecting induced magnetic field, wherein they considered a realistic pulsed pressure gradient instead of constant pressure gradient.

All these studies are restricted in the consideration of electrically nonconducting vessels wall. They argued that the interaction of magnetic field with blood flow induces electric current distribution inside the vessel and formed lobes with positive and negative charges, which in turn causes reduction in blood flow rate. Kinouchi et al. [16] assumed that the electrical conductivity of the arterial walls are nonzero and it will therefore transmit electric current from blood into the surrounding tissues. However, in a physiological situation, the typical electrical conductivity of the tissues is much smaller than the electrical conductivity of blood.

It is now well known that the biological effects on patient when exposed to a high static magnetic field in MRI are still somehow unclear to the biomedical engineers and scientists because of its lack of information. Nowadays most of the MRI machines used $1.5 \mathrm{~T}$ as a strength of the magnetic field, which is $10^{5}$ times the earth's magnetic field strength. However, medical scientists/engineers want to increase the magnetic field strength from $1.5 \mathrm{~T}$ to $3 \mathrm{~T}$ or more upto $8 \mathrm{~T}$ not only in MRI but also in the hyperthermic treatment of cancer therapy (cf. Raymond et al. [17] and Jekic et al. [18]). When a high static magnetic field is imposed to a human body, it needs to have clear information/conclusion for medical disorders of health such as cardiovascular diseases, determination of blood pressure, flow rate, effects of motion-induced currents, voltage measurements in ECG signals, chemical reaction, and thermal responses. Very clearly, Formica and Silvestri [19] made a literature survey on biological effects of exposure to a magnetic resonance imaging. In MRI, the patient is subjected to a high static magnetic field. The movement of charges in the blood causes magnetohydrodynamic effect that induces a voltage across the vessel. This voltage distorts the T-wave in electrocardiogram (ECG) signal of the patient. In this direction several experimental studies on large animal species such as monkey, baboons, and dogs were made by Tenforde [20, 21] and Tenforde et al. [22]. Similar interaction on humans exposure to a strong magnetic field was observed by Jehenson et al. [23]. They observed that there is no such significant effects on either cardiac function or the dynamics of blood flow by the application of magnetic field of strength upto $2 \mathrm{~T}$. In a separate experimental study of Chakeres et al. [24] on several men and women of ages between 24 and 53 years exposure to a static magnetic field of strength $8 \mathrm{~T}$ concluded that there is no clinically significant changes in vital signs. They noticed that the transient ECG artifacts increase with the increase of magnetic field strength.

Three-dimensional numerical modelling of arterial blood flow interaction with transverse magnetic field is the main concern in this paper. Our aim is to compare the present numerical solutions with those obtained approximate/analytical solutions and to estimate the steady and unsteady entry length in terms of the Hartmann number, Womersley

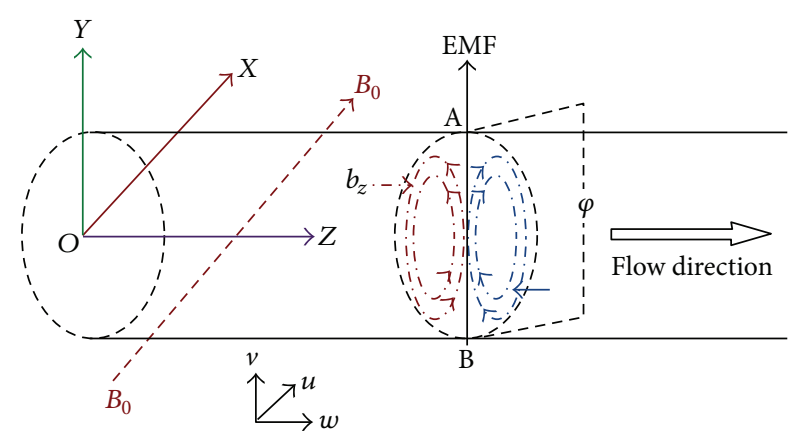

Figure 1: Physical sketch of the problem.

parameter, and the Reynolds number required for numerical simulation in the physiological system. The distribution of current and their directions as well as electric voltage estimation within the artery have been also the topic of this research. Thus the results presented here can be used for the benchmark of most general numerical solutions.

\section{Mathematical Formulation}

2.1. Representation of Elementary Volume. Considering the unsteady flow of a Newtonian, incompressible, and electrically conducting fluid through a straight circular pipe in the presence of an externally applied magnetic field, we assume that the straight circular pipe is of infinite length and an external uniform magnetic field of strength $B_{0}$ is applied along the +ve $x^{\prime}$-axis, perpendicular to the flow direction (+ve $z^{\prime}$-axis). Due to the imposition of external magnetic field, an electromotive force (EMF) is generated in the direction (+ve $y^{\prime}$-axis) perpendicular to both magnetic field and flow directions, inducing a current inside the flow region. Then the total magnetic field assumes in the form $\vec{B}^{\prime}=\vec{B}_{0}+$ $\vec{b}^{\prime}\left(x^{\prime}, y^{\prime}, z^{\prime}, t^{\prime}\right)=B_{0} \hat{i}+\vec{b}^{\prime}$, where $\vec{b}^{\prime}$ denotes the induced magnetic field having components $\left(b_{x^{\prime}}^{\prime}, b_{y^{\prime}}^{\prime}, b_{z^{\prime}}^{\prime}\right)$ and $(\hat{i}, \hat{j}, \widehat{k})$ indicate the unit vectors along the coordinate axes. Let $\vec{V}^{\prime}$ be the velocity vector with components $\left(u^{\prime}, v^{\prime}, w^{\prime}\right)$ along the coordinate axes $\left(x^{\prime}, y^{\prime}, z^{\prime}\right)$ of the circular vessel of radius $a$. Where prime denotes the dimensional quantities of the variables. Figure 1 represents the physical configuration of the flow domain showing the coordinate system, direction of flow field, and applied magnetic and induced magnetic fields. In the figure $\varphi$ denotes the induced voltage to be determined as the potential difference between the electrodes positioned (A and $\mathrm{B}$ ) at the end of the tube diameters perpendicular to the applied magnetic field.

2.2. Governing Equations. The basic governing equations for magnetohydrodynamic (MHD) fluid flow are

$$
\begin{gathered}
\vec{\nabla}^{\prime} \cdot \vec{V}^{\prime}=0 \\
\rho\left(\frac{\partial \vec{V}^{\prime}}{\partial t^{\prime}}+\left(\vec{V}^{\prime} \cdot \vec{\nabla}^{\prime}\right) \vec{V}^{\prime}\right)=-\vec{\nabla}^{\prime} p^{\prime}+\eta \vec{\nabla}^{\prime 2} \vec{V}^{\prime}+\vec{J}^{\prime} \times \vec{B}^{\prime}
\end{gathered}
$$


along with the Maxwell's equations of electromagnetism

$$
\begin{gathered}
\vec{\nabla}^{\prime} \cdot \vec{E}^{\prime}=\frac{\rho_{e}}{\varepsilon} \\
\vec{\nabla}^{\prime} \times \vec{E}^{\prime}=-\frac{\partial \vec{B}^{\prime}}{\partial t^{\prime}} \\
\vec{\nabla}^{\prime} \cdot \vec{B}^{\prime}=0 \\
\vec{\nabla}^{\prime} \times \vec{B}^{\prime}=\mu_{e} \vec{J}^{\prime}
\end{gathered}
$$

and Ohm's law

$$
\vec{J}^{\prime}=\sigma\left[\vec{E}^{\prime}+\vec{V}^{\prime} \times \vec{B}^{\prime}\right]
$$

where $p^{\prime}, t^{\prime}, \rho, \eta, \rho_{e}, \varepsilon, \mu_{e}, \sigma, \vec{J}^{\prime}$, and $\vec{E}^{\prime}$ denote, respectively, the fluid pressure, time, fluid density, dynamic viscosity of the fluid, charge density, vacuum permittivity, magnetic permeability, electrical conductivity, induced current density vector, and induced electric field vector. The last term on the right hand side of (2) represents the electromagnetic force per unit volume due to the interaction of the electric current and the magnetic field.

Following the assumptions (cf. [25]) that the magnetohydrodynamics is distinguished from the study of electromagnetic waves by the time scale, the displacement current is therefore neglected.

Substituting the expression for $\vec{J}^{\prime}$ from (6) in (2) we get

$$
\begin{aligned}
\rho\left(\frac{\partial \vec{V}^{\prime}}{\partial t^{\prime}}+\left(\vec{V}^{\prime} \cdot \vec{\nabla}^{\prime}\right) \vec{V}^{\prime}\right)= & -\vec{\nabla}^{\prime} p^{\prime}+\eta \vec{\nabla}^{\prime 2} \vec{V}^{\prime} \\
& +\frac{1}{\mu_{e}}\left(\vec{\nabla}^{\prime} \times \vec{B}^{\prime}\right) \times \vec{B}^{\prime} .
\end{aligned}
$$

Taking curl of (7) and using (4)-(6), the magnetic induction equation becomes

$$
\frac{\partial \vec{B}^{\prime}}{\partial t^{\prime}}=\vec{\nabla}^{\prime} \times\left(\vec{V}^{\prime} \times \vec{B}^{\prime}\right)+\frac{1}{\sigma \mu_{e}} \vec{\nabla}^{\prime 2} \vec{B}^{\prime}
$$

which yields

$$
\frac{\partial \vec{B}^{\prime}}{\partial t^{\prime}}+\left(\vec{V}^{\prime} \cdot \vec{\nabla}^{\prime}\right) \vec{B}^{\prime}=\left(\vec{B}^{\prime} \cdot \vec{\nabla}^{\prime}\right) \vec{V}^{\prime}+\frac{1}{\sigma \mu_{e}} \vec{\nabla}^{\prime 2} \vec{B}^{\prime} .
$$

Let us introduce the following non-dimensional variables:

$$
\begin{gathered}
\vec{V}(u, v, w)=\frac{\vec{V}^{\prime}}{V_{0}}, \quad p=\frac{p^{\prime}}{\rho V_{0}^{2}}, \quad t=\omega t^{\prime}, \\
\vec{B}=\frac{\vec{B}^{\prime}}{B_{\text {ref }}}=\frac{B_{0} \hat{i}+\vec{b}^{\prime}}{B_{\text {ref }}}, \quad \vec{b}=\frac{\vec{b}^{\prime}}{B_{\text {ref }}}, \\
(x, y, z)=\left(\frac{x^{\prime}}{a}, \frac{y^{\prime}}{a}, \frac{z^{\prime}}{a}\right),
\end{gathered}
$$

where $B_{\text {ref }}=1 / a \sqrt{\eta / \sigma}, \omega$ is the angular frequency, $T$ the time period in cycle is defined by the relation of the form
$T=2 \pi / \omega$, and $V_{0}$ is the mean velocity at the entry section of the tube.

Using these dimensionless variables, (1), (8), and (10) in non-dimensional form may be expressed as

$$
\begin{aligned}
& \vec{\nabla} \cdot \vec{V}=0 \\
& \frac{\alpha^{2}}{\operatorname{Re}} \frac{\partial \vec{V}}{\partial t}+(\vec{V} \cdot \vec{\nabla}) \vec{V}=-\vec{\nabla} p+\frac{1}{\operatorname{Re}} \vec{\nabla}^{2} \vec{V}+\frac{\mathrm{Ha}}{\operatorname{Re} R_{m}}(\vec{\nabla} \times \vec{b}) \\
& \times \hat{i}+\frac{1}{\operatorname{Re} R_{m}}(\vec{\nabla} \times \vec{b}) \times \vec{b}, \\
& \frac{\alpha^{2}}{\operatorname{Re}} \frac{\partial \vec{b}}{\partial t}+(\vec{V} \cdot \vec{\nabla}) \vec{b}=(\vec{b} \cdot \nabla) \vec{V}+\operatorname{Ha}(\hat{i} \cdot \vec{\nabla}) \vec{V}+\frac{1}{R_{m}} \vec{\nabla}^{2} \vec{b}
\end{aligned}
$$

where four non-dimensional parameters that appeared in (13) are defined as $\mathrm{Ha}=B_{0} a \sqrt{\sigma / \eta}$, the Hartmann number (is the ratio of electromagnetic force to the viscous force); $R_{m}=a V_{0} \mu_{e} \sigma$, the magnetic Reynolds number (estimates the effects of magnetic advection to magnetic diffusion); $\mathrm{Re}=$ $a \rho V_{0} / \eta$, the Reynolds number (ratio of the fluid convection to viscous forces), and the Womersley number $\alpha=a \sqrt{\omega / v}$ with frequency $\omega$ and the kinematic viscosity $v$ represents the relative importance of the unsteady inertia to viscous forces.

It is worthwhile to mention here that the magnetic field is normalized by the material property of the fluid such as the dynamic viscosity $\eta$, electrical conductivity $\sigma$, and the tube radius $a$. These quantities are fixed with respect to the applied magnetic field strength. It may, therefore, provide correct formulation of the magnetohydrodynamic equations. This concept is already mentioned by several investigators $[1,16,26]$ in different forms. However, the rest of the studies $[10,12,27]$ on the topic are based on the normalization of magnetic field by the strength of applied external magnetic field which may lead to contradiction when studying varying applied magnetic field. In order to do so, our normalization of magnetic field makes the Hartmann number a dimensionless external magnetic field $\left(\mathrm{Ha}=B_{0} / B_{\text {ref }}\right)$.

2.3. Boundary Conditions. The governing equations (12)-(13) for magnetohydrodynamic flow of blood presented in the preceding section must be solved subjected to the appropriate boundary conditions. For the velocity components, we imposed $w^{\prime}=V_{0}$ for steady flow and $w^{\prime}=V_{0}\left[1+\sin \left(\omega t^{\prime}+\right.\right.$ $(3 \pi / 2))$ ] for unsteady flow along with $u^{\prime}=v^{\prime}=0$ at the entry section of the straight tube. The sinusoidal wave form is used, so that the duration of the net zero flow diminishes. The choice of this cardiac signal is considerably dependant on the zero flow at time $t=0$ and reaches its peak value equal to 2 in order to maintain the net flow rate $=1$. The boundary conditions in non-dimensional form for both the cases are listed in Table 1.

The usual no-slip velocity condition $(u=v=w=0)$ is used at the vessel wall with the assumption of the straight circular tube being rigid. For unsteady flow, the symmetric boundary conditions imposed in the XOZ and YOZ plane as $(\widehat{n} \cdot \vec{\nabla}) \vec{V}=\overrightarrow{0}$, where $\widehat{n}$ being the unit vector normal to the 
TABLE 1: Boundary conditions at a glance.

\begin{tabular}{|c|c|c|c|c|}
\hline Flow condition & Entry (inlet) section & Exit (outlet) section & Rigid wall & $\begin{array}{c}\text { Symmetric boundary XOZ } \\
\text { and YOZ plane }\end{array}$ \\
\hline Steady flow & $w=1, u=v \underset{\overrightarrow{0}}{=} 0,(\widehat{n} \cdot \vec{\nabla}) \vec{b}=$ & $\begin{array}{c}p=0 \text { with no viscous } \\
\text { stress, }(\vec{n} \cdot \vec{\nabla}) \vec{b}=\overrightarrow{0}\end{array}$ & $u=v=w=0, \vec{b}=\overrightarrow{0}$ & Not applicable \\
\hline Unsteady flow & $\begin{array}{c}w=[1+\sin (t+3 \pi / 2)] \\
u=v=0,(\widehat{n} \cdot \vec{\nabla}) \vec{b}=\overrightarrow{0}\end{array}$ & $\begin{array}{l}p=0 \text { with no viscous } \\
\text { stress, }(\hat{n} \cdot \vec{\nabla}) \vec{b}=\overrightarrow{0}\end{array}$ & $u=v=w=0, \vec{b}=\overrightarrow{0}$ & $\begin{array}{c}\text { For both the planes: } \\
(\widehat{n} \cdot \vec{\nabla}) \vec{V}=\overrightarrow{0} \text { and at YOZ: } \\
\vec{b}=\overrightarrow{0} \text { at } \mathrm{XOZ}:(\vec{n} \cdot \vec{\nabla}) \vec{b}=\overrightarrow{0}\end{array}$ \\
\hline
\end{tabular}

considered surface. In addition to the flow-field information is the pressure that plays an important role, since the blood is directly supplied from the right heart and pumped out with a pulsatile pressure gradient by the contraction and expansion of the muscle heart. In our model, no pressure is imposed at the inlet of the tube, since it is directly dependant on the inlet flow rate. However, to maintain small resistance, the cross-sectional average pressure $p$ at the outlet is set to zero. Therefore, the fluid pressure depend on the axial position $z$, time, and magnetic field.

2.4. Derivation of Induced Current Density, Electric Field, and Voltage. After having determined numerically the velocity and induced magnetic field components, the numerical values to the induced current, electric field, and voltage can be obtained by the following expressions.

From (6), we can derive current density vector as

$$
\vec{J}^{\prime}=\frac{1}{\mu_{e}} \nabla^{\prime} \times \vec{B}^{\prime}=\frac{1}{\mu_{e}} \nabla^{\prime} \times \vec{b} .
$$

Using the dimensionless variables defined in (11), we write

$$
\vec{J}=\frac{\vec{J}^{\prime}}{J_{0}} \nabla \times \vec{b}
$$

where $J_{0}=1 / \mu_{e} a^{2} \sqrt{\eta / \sigma}$.

The magnetohydrodynamic interaction gives rise to an induced electric field $\vec{E}$, which can be obtained from Ohm's law (7) in the following form:

$$
\vec{E} \frac{\vec{J}^{\prime}}{\sigma}-\vec{V}^{\prime} \times \vec{B}^{\prime}
$$

and its expression in non-dimensional form becomes

$$
\vec{E}=\frac{\vec{E}^{\prime}}{E_{0}}=\frac{1}{R_{m}} \vec{J}-\vec{V} \times(\mathrm{Ha} \hat{i}+\vec{b}),
$$

where $E_{0}=V_{0} B_{\text {ref }}$ in which magnitude of the referred field is $B_{\text {ref }}=1 / a \sqrt{\eta / \sigma}$.

Therefore, the non-dimensional blood flow potential difference (also known as induced voltage) between the electrodes positioned at the end of the diameter of each cross section of the tube in $y$-direction can be obtained by the line integration of $E_{y}$ as follows:

$$
\begin{aligned}
\Phi & =\frac{\Phi^{\prime}}{E_{0} a}=-\int_{-1}^{1} E_{y} d y \\
& =-\int_{-1}^{1}\left\{\frac{1}{R_{m}} J_{y}-w\left(\mathrm{Ha}+b_{x}\right)+u b_{z}\right\} d y .
\end{aligned}
$$

However, for the flow symmetry, in the present study the induced voltage is obtained as

$$
\begin{aligned}
\Phi & =\frac{\Phi^{\prime}}{E_{0} a}=-2 \int_{0}^{1} E_{y} d y \\
& =-2 \int_{0}^{1}\left\{\frac{1}{R_{m}} J_{y}-w\left(\mathrm{Ha}+b_{x}\right)+u b_{z}\right\} d y .
\end{aligned}
$$

It has also been observed by Tenforde [21] that the largest magnetically induced voltage occurs during pulsatile blood flow in the aorta.

\section{Numerical Methods and Mesh Refinements}

Direct numerical simulations were performed with Comsol Multiphysics (version 3.5a) software to solve the magnetohydrodynamic (MHD) partial differential equations. Comsol Multiphysics software provides us to implement finite element meshing to create a three-dimensional model of blood vessel. The convergence of the numerical solutions has been verified by mesh refinement. In finite element method, the easy process of mesh refinement is the use of different element size (ES) in non-dimensional form. The decrease of element size (ES) increases the number of elements (NE) as well as the degrees of freedom (DOF) and thereby increases the accuracy of the numerical solutions. In Table 2, we studied the mesh refinement in four different cases with different maximum element size and compared the solutions for axial velocity, as it is the main component of the flow field.

Table 3 illustrates how the solution is changed with different element size. The differences of the solutions between Case-I and Case-II, Case-II, and Case-III, and Case-III and Case-IV have been presented in three different column of Table 3 for Hartmann number $\mathrm{Ha}=2$ and $\mathrm{Ha}=10$. It is observed that the discrepancy between the solutions obtained in Case-III \& Case-IV is limited in $0.01 \%$. If we consider CaseIII, our solution reaches up to 99.99 percent of the correct 
TABLE 2: Mesh statistics studied in four different cases for a quarter part of the tube.

\begin{tabular}{lccccc}
\hline Case study & $\begin{array}{c}\text { Maximum element } \\
\text { size (ES) } \\
\text { (dimensionless) }\end{array}$ & $\begin{array}{c}\text { Number of } \\
\text { elements (NE) }\end{array}$ & $\begin{array}{c}\text { Number of degrees of } \\
\text { freedom (DOF) }\end{array}$ & $\begin{array}{c}\text { Number of triangular } \\
\text { element at the entry } \\
\text { section }\end{array}$ & $\begin{array}{c}\text { Number of triangular } \\
\text { element at the surface of } \\
\text { the vessel wall }\end{array}$ \\
\hline Case-I & 0.30 & 46568 & 466982 & 38 & 6528 \\
Case-II & 0.20 & 163594 & 1538336 & 80 & 13486 \\
Case-III & 0.18 & 226680 & 2102756 & 89 & 17366 \\
Case-IV & 0.15 & 394192 & 3577693 & 125 & 25696 \\
\hline
\end{tabular}

TABle 3: Absolute error in the axial velocity $w(x, 0.5,90)$ for four different cases when $\operatorname{Re}=300$.

\begin{tabular}{ccccccc}
\hline$x$ & & $\mathrm{Ha}=2$ & & \multicolumn{1}{c}{$\mathrm{Ha}=10$} \\
& Case-I and Case-II & Case-II and Case-III & Case-III and Case-IV & Case-I and Case-II & Case-II and Case-III & Case-III and Case-IV \\
\hline 0.0 & $5 \times 10^{-5}$ & $4 \times 10^{-5}$ & $1 \times 10^{-5}$ & $5 \times 10^{-3}$ & $4 \times 10^{-5}$ & $1 \times 10^{-5}$ \\
0.2 & $2 \times 10^{-4}$ & $4 \times 10^{-5}$ & $2 \times 10^{-5}$ & $3 \times 10^{-3}$ & $5 \times 10^{-6}$ & $3 \times 10^{-6}$ \\
0.4 & $3 \times 10^{-4}$ & $6 \times 10^{-5}$ & $1 \times 10^{-5}$ & $1 \times 10^{-3}$ & $2 \times 10^{-4}$ & $1 \times 10^{-5}$ \\
0.5 & $2 \times 10^{-4}$ & $6 \times 10^{-5}$ & $1 \times 10^{-5}$ & $4 \times 10^{-3}$ & $9 \times 10^{-4}$ & $4 \times 10^{-5}$ \\
0.6 & $8 \times 10^{-5}$ & $1 \times 10^{-5}$ & $3 \times 10^{-5}$ & $1 \times 10^{-2}$ & $1 \times 10^{-3}$ & $1 \times 10^{-4}$ \\
0.8 & $6 \times 10^{-4}$ & $8 \times 10^{-5}$ & $3 \times 10^{-5}$ & $8 \times 10^{-3}$ & $1 \times 10^{-3}$ & $2 \times 10^{-4}$ \\
\hline
\end{tabular}

result. Thus, the choice of Case-III mesh refinement gives less CPU time as well as being economic in memory space. It is observed from Table 3 that for higher values of the Hartmann number Ha needs extra fine mesh to get desired accuracy. However, we observed that the results are more accurate near the central line of the tube rather than in the boundary layer.

\section{Computational Results and Discussion}

In this section, we have presented the computational results obtained from the above mentioned numerical model. In order to illustrate the complete numerical solutions, the following set of values of the rheological properties of blood in the case of aorta have been used as per $\mathrm{He}$ and $\mathrm{Ku}$ [9], Kinouchi et al. [16], and Abi-Abdallah et al. [12]:

$$
\begin{gathered}
\eta=0.004 \mathrm{~Pa} \cdot \mathrm{s} ; \quad \rho=1050 \frac{\mathrm{kg}}{\mathrm{m}^{3}} \\
\sigma=0.5 \frac{\mathrm{S}}{\mathrm{m}} ; \quad a=10^{-2} \mathrm{~m} ; \quad \mu_{e}=4 \pi 10^{-7} \frac{\mathrm{H}}{\mathrm{m}} .
\end{gathered}
$$

These data provides the relation between Hartmann number $\mathrm{Ha}$ and magnetic field strength $B_{0}$ (in Tesla) as $B_{0}=$ 9Ha. In the present study, the values of the Hartmann number, Reynolds number, magnetic Reynolds number, and the Womersley parameters vary between the range as $\mathrm{Ha} \in$ $[0,10]$; Re $\in\left[3 \times 10^{-1}, 3 \times 10^{2}\right] ; R_{m} \in O\left(10^{-9}\right) ; \alpha \in[2,10]$. For hemodynamical flows in aorta, the range of values of $\mathrm{Ha}(0 \leq \mathrm{Ha} \leq 2)$ has been chosen as an applicability of reference value.

With an aim to test the accuracy of our numerical results, the comparison have been made with the analytical solutions for steady case in the fully developed region. Figure 2 depicts the comparison of axial velocity for $\mathrm{Ha}=1$ and 10 and shows excellent agreement with those of Fabri and Siestrunck [26].

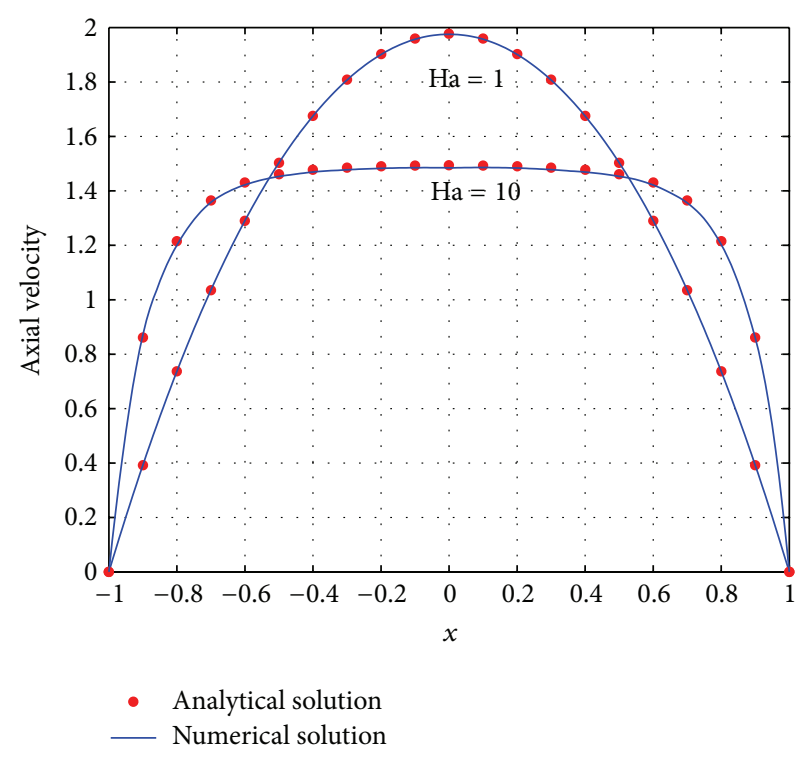

Figure 2: Comparison of axial velocity profile in the developed region with the analytical solutions of Fabri and Siestrunck [26] for $\mathrm{Ha}=1$ and $\mathrm{Ha}=10$.

4.1. Entry Length for Steady Flow Development. In our numerical model, we considered a cylindrical tube of length 100 unit in non-dimensional form. We used cartesian coordinate system $(x, y, z)$ in which the axis of the tube lies in the $z$ direction. In this model we used uniform velocity profile at the entrance, that is, $w=1$ at $z=0$. For the sake of comparison we have also considered the cases when the entry velocity are assumed to be parabolic and reverse parabolic profile, respectively. Due to the choice of different entry profiles at the entrance and when the fluid starts to move, the no-slip velocity $u=v=w=0$ at the wall comes into effect. 


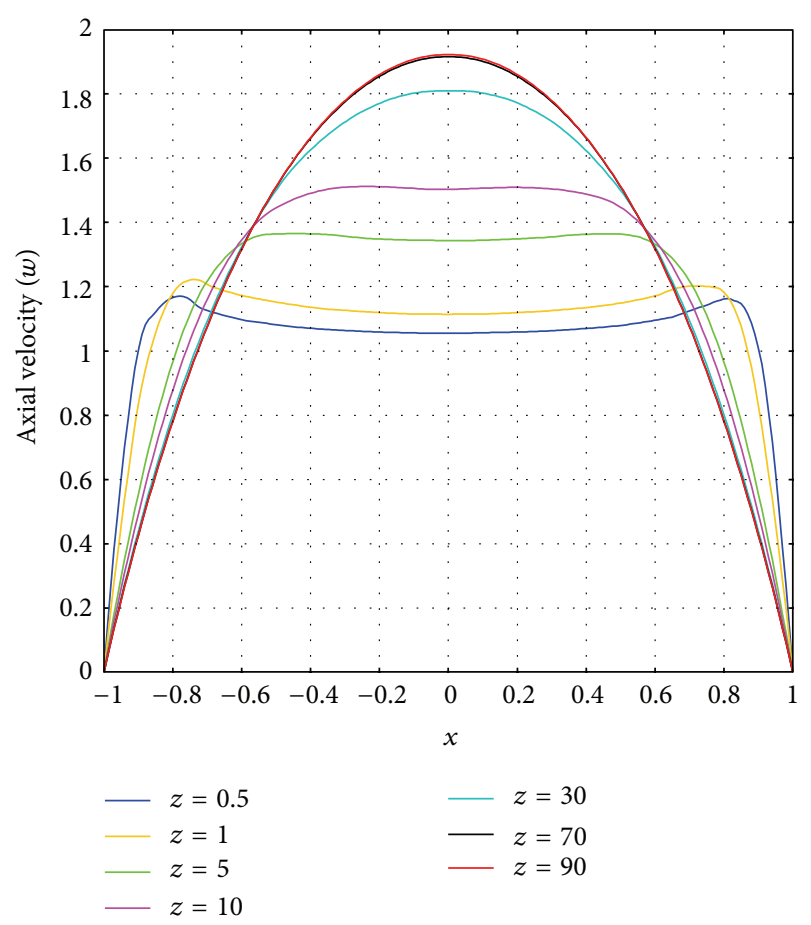

FIgURE 3: Steady flow development of axial velocity $w$, at different axial position in the presence of magnetic field, when $\mathrm{Re}=300$ and $\mathrm{Ha}=2$.

The flow adjacent to the wall decelerates continuously. Due to the profile adjustment in $z$-direction as shown in Figure 3, the pressure will decrease gradually along $z$-direction and a thin boundary layer is formed round the walls of the pipe. It is observed that the axial velocity does not have any significant change beyond certain distance and that other flow quantities except pressure are independent of axial position.

Figure 4 shows the estimation of entry length in terms of the Reynolds number Re when no magnetic field is applied $(\mathrm{Ha}=0)$. We measured the approximate entry length $L_{e}$ from our numerical solution by restricting the error within $1 \%$. The process of the measurement of entry length $z$, is the nondimensional distance along the axis where the central line axial velocity reaches $99 \%$ of its fully developed value (exactly following the method of $\mathrm{He}$ and $\mathrm{Ku}$ [9]). The estimated numerical values are closely corresponding to the relation of the form $L_{e} \cong 0.25 \mathrm{Re}$ as shown in Figure 4 with solid line.

It is worthwhile to mention here that when the entry length is normalized by the diameter of the tube and the Reynolds number is defined in terms of the diameter, then the entry length $L_{e}$ becomes $L_{e} \cong 0.06 \mathrm{Re}$, which is exactly following from the relation established by experimental studies (cf. Joulie [28]; Comolet [29]; Janna [30]).

The steady entrance length in terms of the Reynolds number Re and the Hartmann number Ha has been obtained from our present numerical simulation. We have also theoretically estimated the following function with the help of general curve fitting technique. This approximate entry length corresponds to our numerical data within a minimum

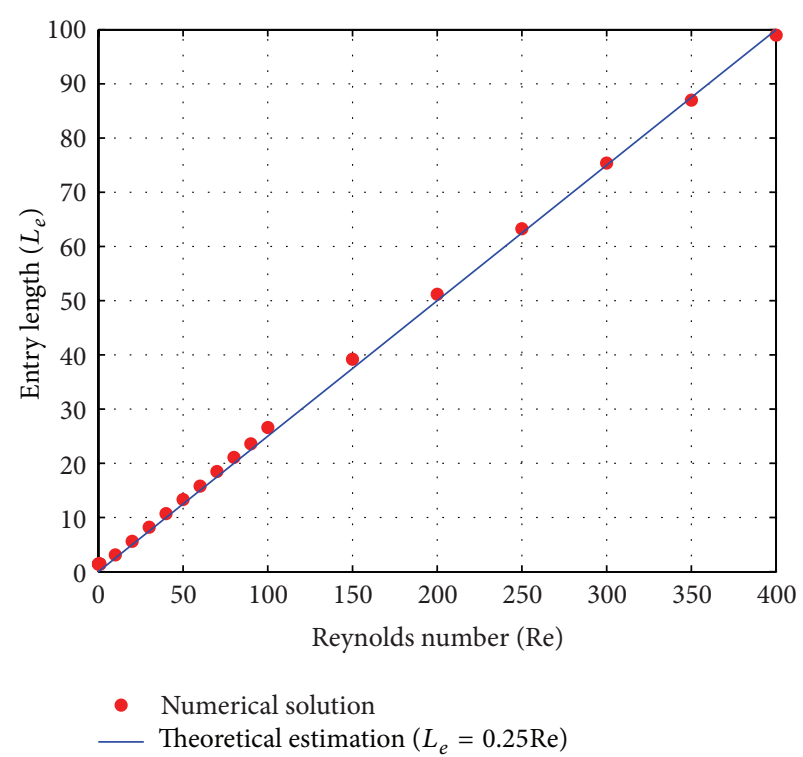

FIGURE 4: Steady entrance length $L_{e}$ versus Reynolds number Re without applying magnetic field $(\mathrm{Ha}=0)$.

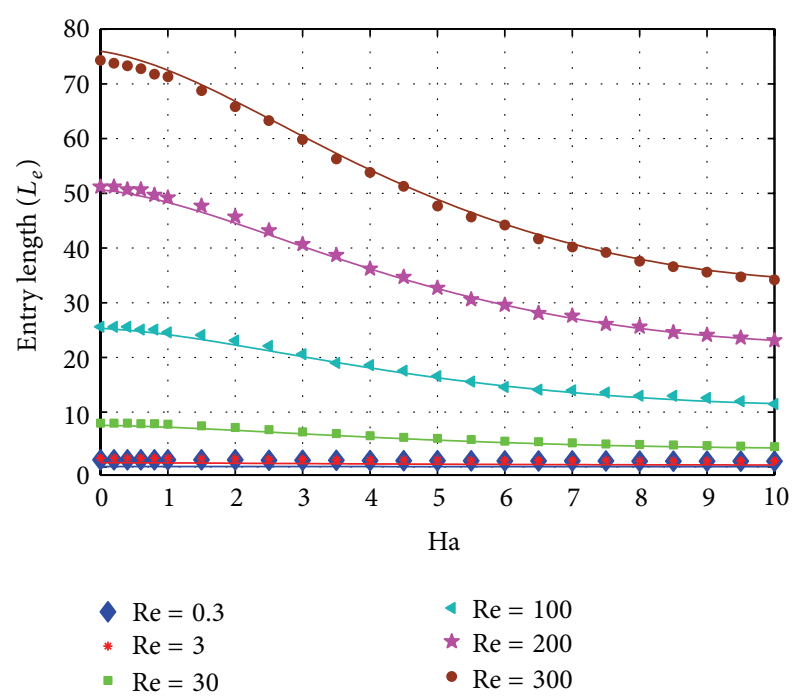

FIGURE 5: Steady entrance length in terms of the Hartmann number $\mathrm{Ha}$ and Reynolds number Re. (Entry length indicated by points obtained from numerical solutions and solid lines represent the theoretical approximation).

range of error as shown in Figure 5, represented by the solid lines:

$$
L_{e} \cong \frac{0.25 \mathrm{Re}}{1+0.024 \mathrm{Ha}+0.025 \mathrm{Ha}^{2}-0.0016 \mathrm{Ha}^{3}} .
$$

For higher values of the Reynolds number Re, the entry length decreases strongly with Hartmann number $\mathrm{Ha}$, while for the lower values of Re the rate of decrease of entry length is insignificant.

Figure 6 depicts the variation of axial velocity profiles in the developed region with different Hartmann number Ha. It is observed that we expect the velocity in the central 


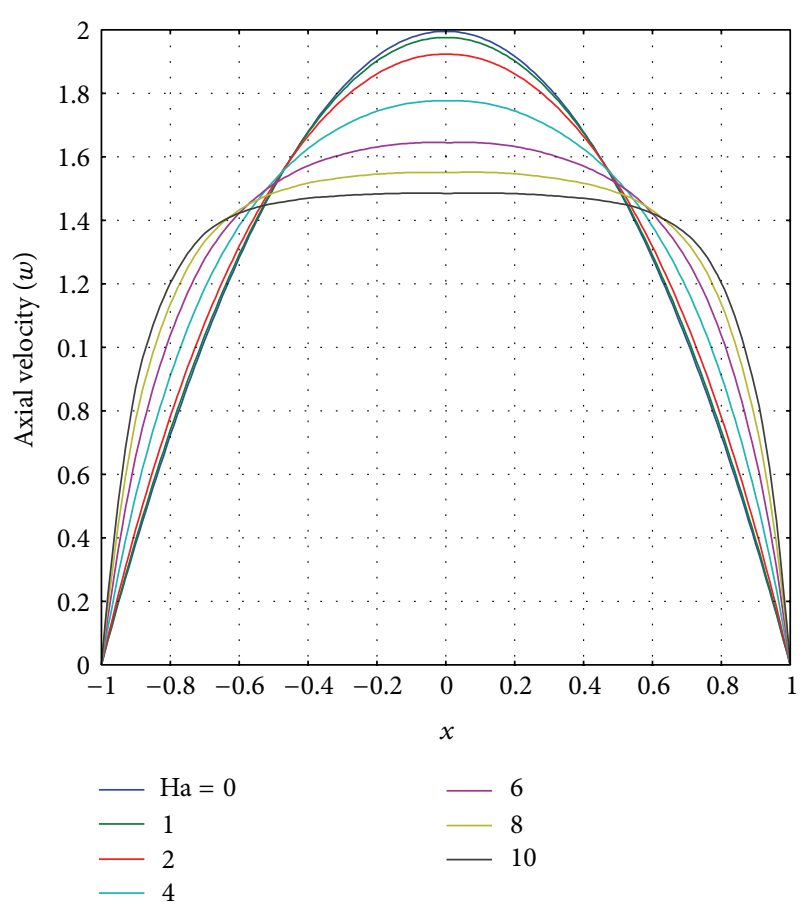

Figure 6: Axial velocity profile for different values of the Hartmann number Ha, when the flow is fully developed in steady case with $\mathrm{Re}=$ 300 .

region to decrease with $\mathrm{Ha}$, while near the vessel wall it has an accelerating effect. However, the mean flow rate throughout the tube remains constant. The reason behind this fact is the pressure gradient, which increases with Hartmann number Ha. Moreover, the Lorentz force that arises due to the application of magnetic field strength plays a vital role for decreasing the main part of the velocity component $w$. Since the axial velocity at the central line of the vessel decreases with the increase of Hartmann number Ha and the entry length depends upon the central line axial velocity, entry length also decreases with the strength of the applied magnetic field.

4.2. Unsteady Entry Length. The unsteady entrance length for physiological system is very important, which has enormous effect than steady entrance length. Few researchers have already determined unsteady entry lengths numerically as well as experimentally. But there is no information available in the scientific literature about the entry lengths for unsteady flow even in the steady flow in magnetic environment. The idea of entrance length is very important to people who are interested in blood flow simulation during MRI process in different physiological situations. For the sake of comparison the results are presented within a time period $T=2 \pi$ in any cycle for different Womersley parameter $\alpha$.

The variation of unsteady entrance length within a period of time (in the 5th cycle) for different Womersley parameter $\alpha$ is shown in Figure 7. It reveals that for lower value of the Womersley parameter $\alpha$, the average entrance length directly follows from the steady entrance length. The maximum entrance length as well as the amplitude of oscillation

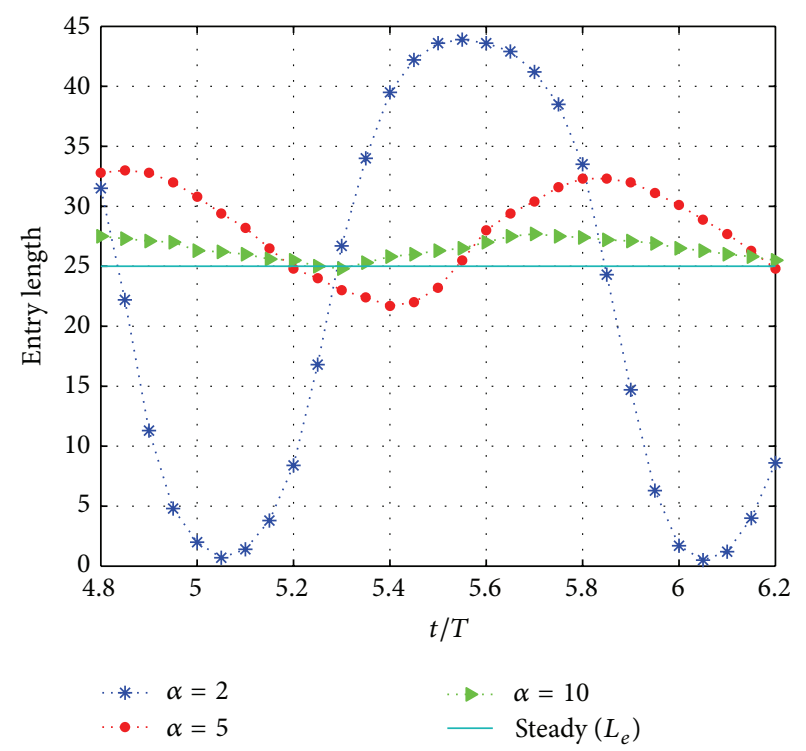

FIGURE 7: Unsteady entrance length with time for different Womersley number $\alpha$, when $\mathrm{Re}=100$ and $\mathrm{Ha}=0.5$ (where the solid line represents the steady entrance length).

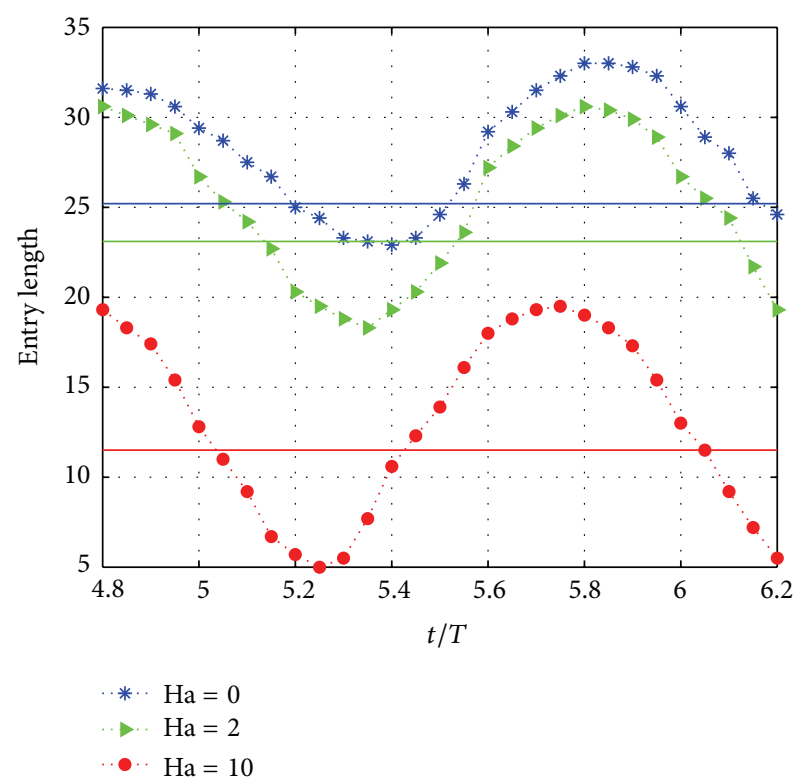

FIGURE 8: Unsteady entrance length with time for different Hartmann number $\mathrm{Ha}$, when $\mathrm{Re}=100$ and $\alpha=5$. (Solid lines represent the corresponding steady entrance length).

decreases with the increase of Womersley parameter $\alpha$. It is important to note that the phase lag occurs significantly with $\alpha$. In the present study, the non-dimensional unsteady entrance length varied between $0.21 \mathrm{Re}$ and $0.33 \mathrm{Re}$, when $\alpha=5$ and $\mathrm{Ha}=0.5$. The unsteady entrance length for different values of the Hartmann number $\mathrm{Ha}$ is presented in Figure 8 . The unsteady entrance length is found to oscillate with time. The unsteady entrance length is also decreased with Hartmann number $\mathrm{Ha}$ as seen in the case of steady flow. The phase difference in entry length is also observed with 


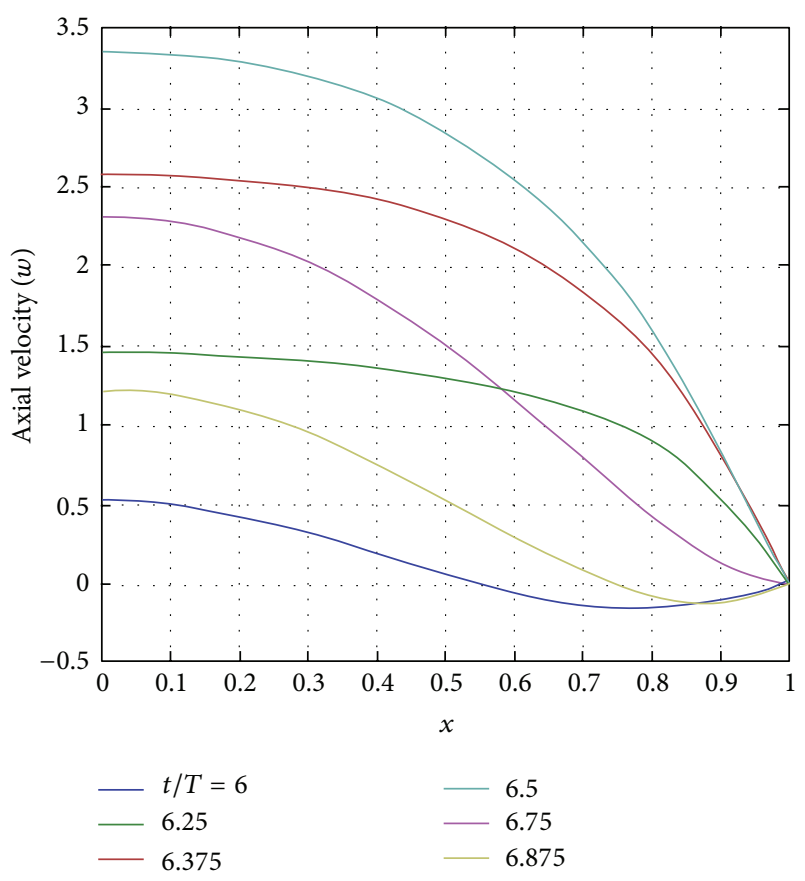

FIGURE 9: Variation of unsteady velocity profiles at different time within a period (6th cycle) for $\mathrm{Re}=100$ and $\mathrm{Ha}=2, \alpha=5$.

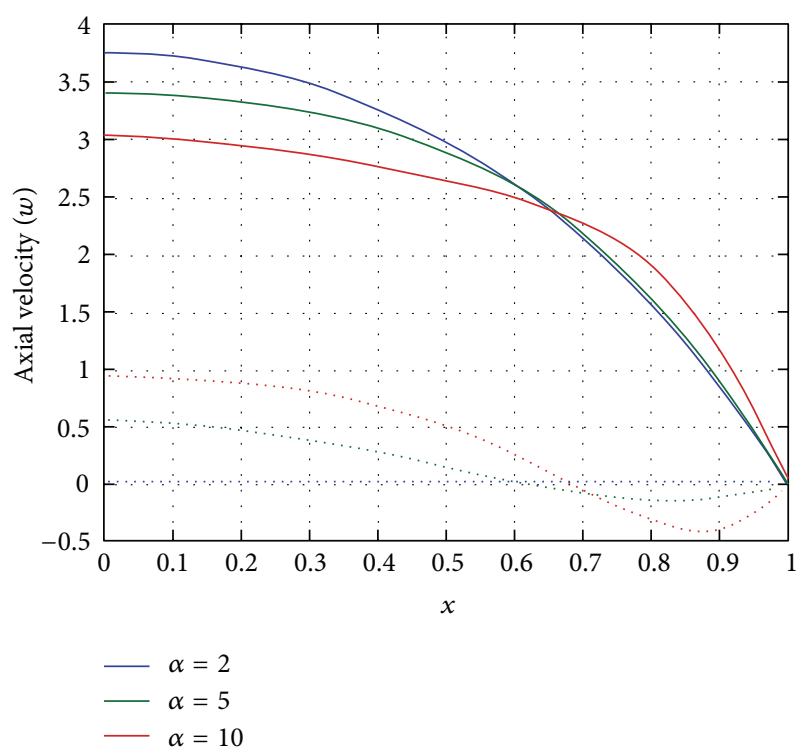

FIGURE 10: Variation of unsteady axial velocity profile at the peak (solid lines) and minimal (dotted lines) velocity for different Womersley parameter $\alpha$, with $\mathrm{Re}=100$ and $\mathrm{Ha}=2$.

the variation of Hartmann number Ha. Thus, it is important to conclude that the average unsteady entrance length is approximately equal to that of the steady entrance length.

Figures 9-11 illustrate the variation of axial velocity profiles with different time in cycle, Womersley parameter $\alpha$, and the Hartmann number Ha. From Figure 9 we observed that the axial velocity oscillates with time in which the peak velocity occurs at $t / T=6.5$ and the minimal value at $t / T=6$

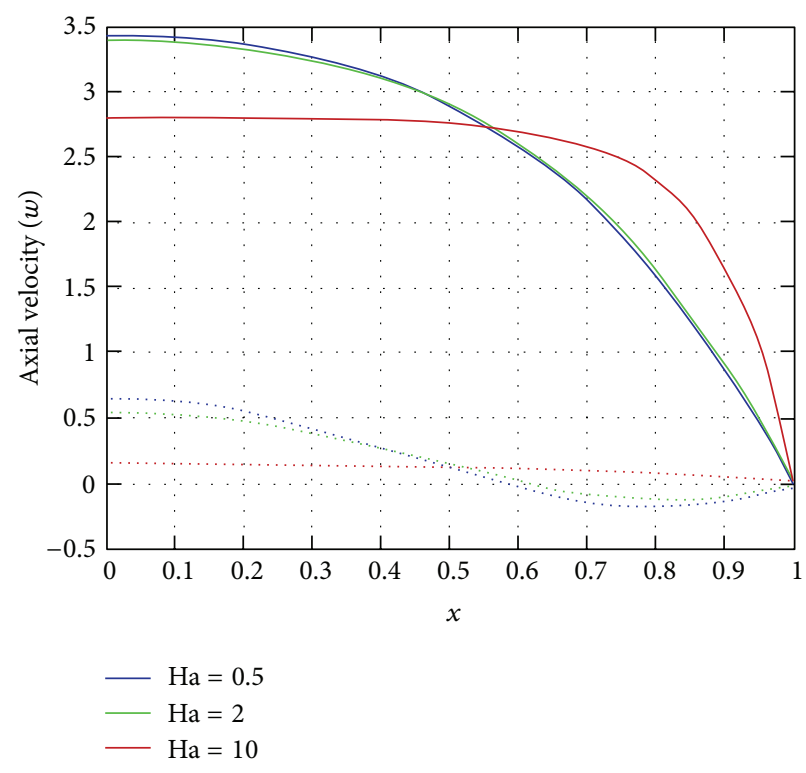

FIGURE 11: Variation of unsteady axial velocity profile at the peak (solid lines) and minimal (dotted lines) velocity for different Hartmann number $\mathrm{Ha}$, with $\mathrm{Re}=100$ and $\alpha=5$.

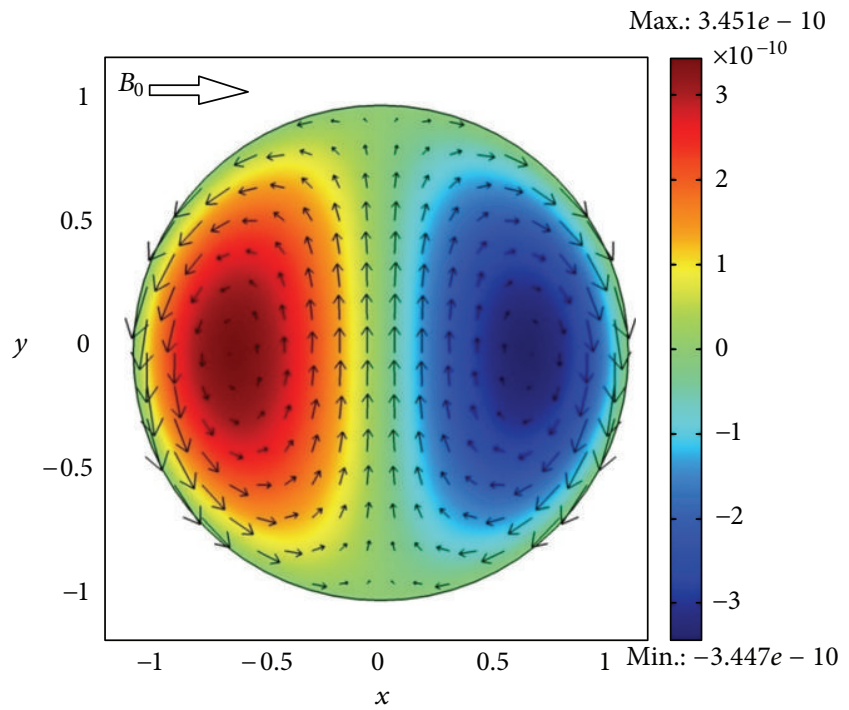

FIGURE 12: Surface plot with color represents the axial induced magnetic field and arrow plot indicates the induced current density in steady case for $\mathrm{Re}=300, \mathrm{Ha}=2$.

or 7 (in the sixth cycle). The flow reversal takes place in the boundary layer. The boundary layer thickness depends on the Womersley parameter $\alpha$ as shown in Figure 10. The unsteady boundary layer thickness decreases with increasing the Womersley parameter $\alpha$. However, the maximum negative value is observed in the case of higher values of $\alpha$. Figure 11 depicts the variation of unsteady velocity profiles for different Hartmann number Ha. It is observed that the peak value of the velocity directly follows the same variation as seen in the steady flow, while in the case of minimal value the trend is reversed. It is interesting to note that the flow 


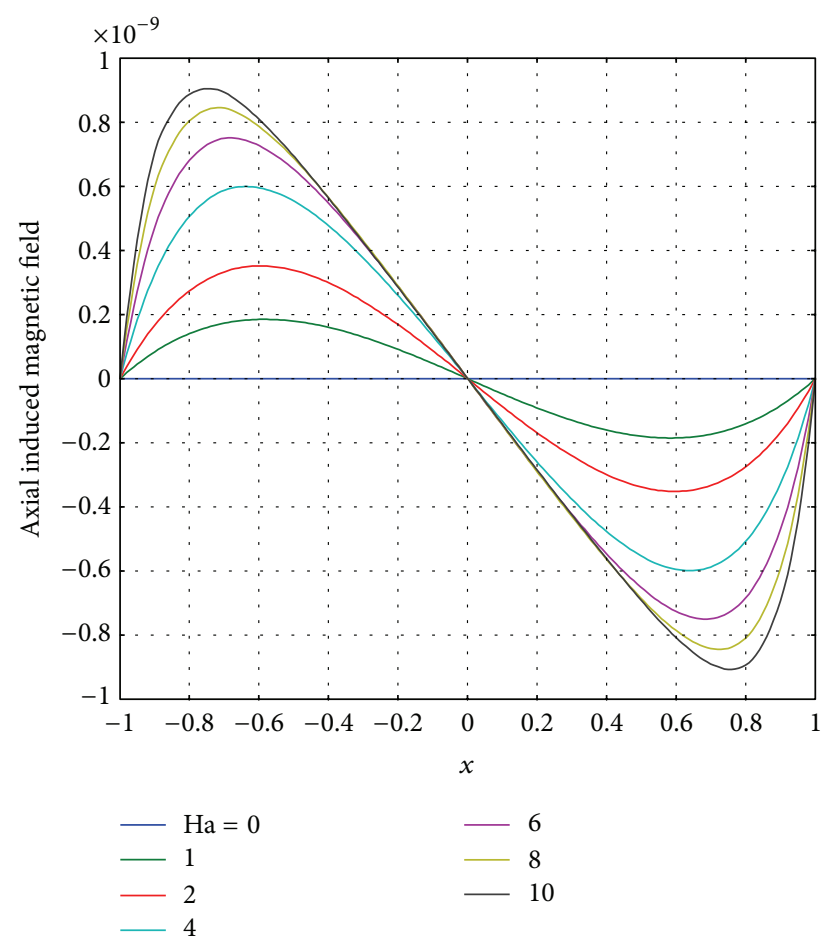

FIGURE 13: Axial induced magnetic field profiles for different Hartmann number $\mathrm{Ha}$ in steady case for $\mathrm{Re}=300$.

reversal can be eliminated with applying strong magnetic field. For higher values of $\mathrm{Ha}$, for example, when $\mathrm{Ha}=10$, the flow reversal is completely removed. However, in the range of values of $\mathrm{Ha}$ for physiological flow, the flow reversal cannot be completely removed, but it is possible to be eliminated with a considerable extent.

4.3. Effect of Induced Magnetic Field. The consideration of induced magnetic field provides better understanding of the studies of arterial blood flow in magnetic environment. Therefore, it is important to characterize the effect of induced magnetic field and to know its behavior with different applied magnetic field strength. The colour plot in Figure 12 presents axial induced magnetic field in the fully developed region of the tube, while the arrow indicates the direction of current flows. The surface plots of axial induced magnetic fields present two lobes on each side of the main current line along $y$-direction. One of these two lobes of axial induced magnetic field circulating in the same direction as the direction of flow, while the other circulates opposite to the flow direction. It is observed that the current is circulating along the central $y$-direction perpendicular to the direction of flow and applied magnetic field as we expect theoretically from (7). Once the current reaches near the wall of the vessel, it moved downwards and formed lobes. This fact lies in the consideration of nonconducting vessel walls which force them to recirculate within the vessel. Figure 13 reveals that the magnitude of induced magnetic field increases with increasing values of Hartmann number Ha. It shows that the magnitude of induced magnetic field increases with the

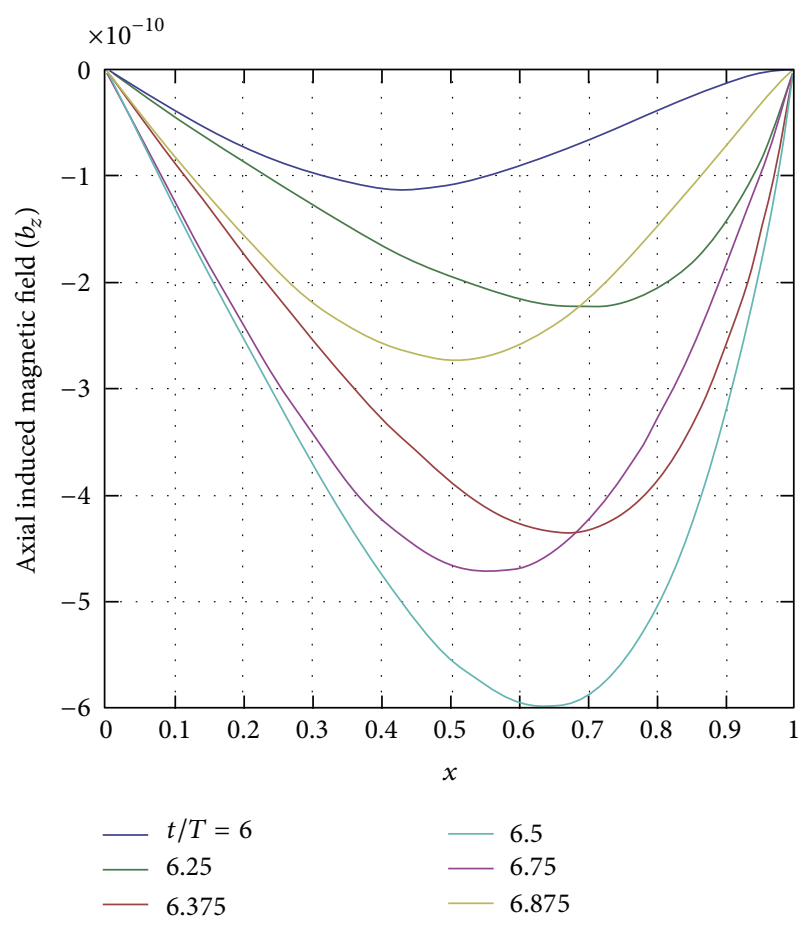

FIgURE 14: Axial induced magnetic field profiles with different time in a period (6th cycle) for $\mathrm{Re}=100, \alpha=5$ and $\mathrm{Ha}=2$.

increase of magnetic field strength, while it is zero in the absence of magnetic field $(\mathrm{Ha}=0)$. It is important to note that as Ha increases, the core region of recirculation of currents is shifted to the vessel walls and causes more flattening of the axial velocity in the core region. This variation is observed asymptotically with higher and increasing values of $\mathrm{Ha}$.

Figures 14 and 15 illustrate the axial induced magnetic field profiles in unsteady case. The results presented in Figure 14 for different instants of time within one period, where the peak value is observed in the middle of a period. The magnitude of the induced magnetic field oscillates and has occurrence of different phase lag with time. We observe that during diastolic phase, the current circulates near the central line, whereas during systolic phase the core region of circulation moves toward the vessel walls. The effect of Womersley parameter $\alpha$ on induced magnetic field is also observed shown in Figure 15. From this result we notice that the magnitude of the induced magnetic field decreases with the increase of $\alpha$. The increase of unsteadiness implying thickening of unsteady boundary layer causes flattening of the core uniform velocity. This observation is also coherent with Hartmann number as shown in Figures 10 and 11. Thus we conclude that there exist proportional effect of Hartmann number and the unsteadiness on the axial induced magnetic field and thereby causes retardation of axial velocity.

4.4. Role of Induced Electric Field and Voltage. The electromagnetic field is described by Maxwell's equations of electromagnetism for which the electric field $\vec{E}$ can be determined from Ohm's law as $\vec{E}=\left(1 / R_{m}\right) \vec{\nabla} \times \vec{b}-\vec{V} \times($ Ha $\vec{i}+\vec{b})$ 


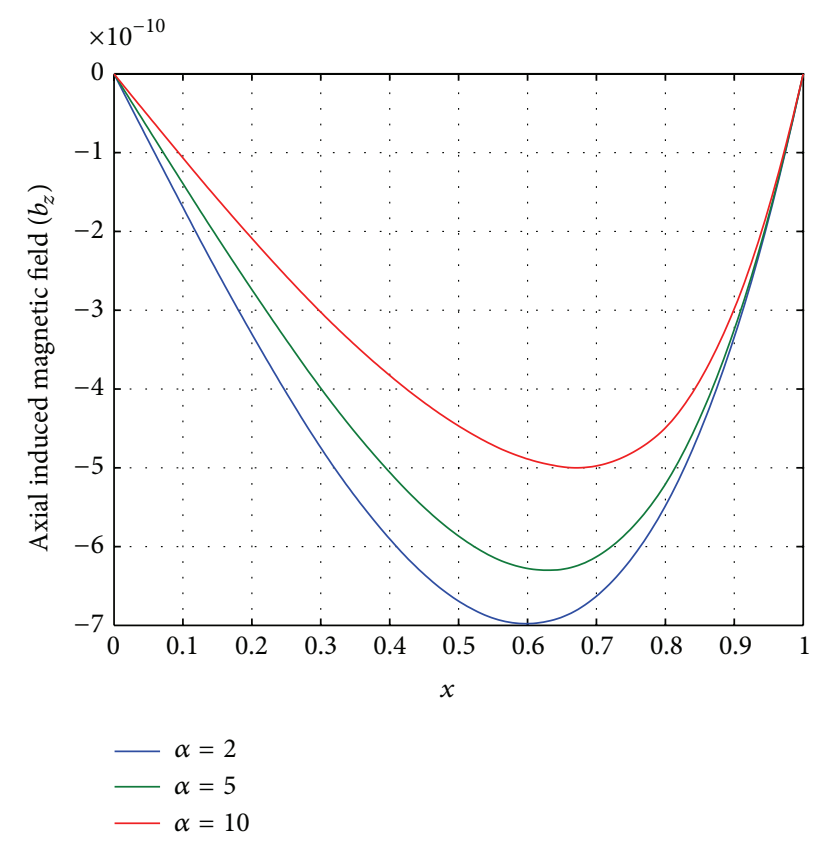

Figure 15: Axial induced magnetic field profiles for different Womersley parameter $\alpha$ during its peak value with $\mathrm{Re}=100$ and $\mathrm{Ha}=2$.

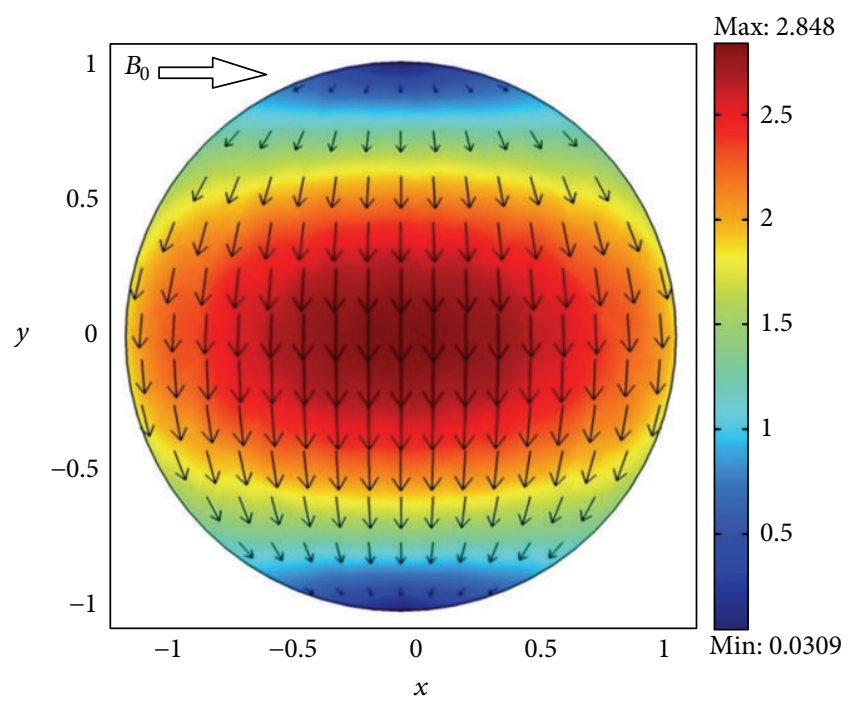

FIGURE 16: The surface plot shown in color is the magnitude of the electric field and arrow plots indicate the direction of induced electric field. The plot is showing in steady case in the far downstream for $\mathrm{Re}=300$ and $\mathrm{Ha}=2$.

(cf. (15) and (17)). Thus it is obvious that the electric field is predominantly dependant on the applied magnetic field and the fluid velocity. The main component of induced electric field is $E_{y}$, which moves along opposite to the current density from positive direction to negative direction as shown in Figure 16. The color plots indicate the magnitude of electric field $|E|=\sqrt{E_{x}^{2}+E_{y}^{2}+E_{z}^{2}}$. The magnitude of the electric field is observed to be maximum at the central region. This confirms that the Lorentz force is strongly active at the central

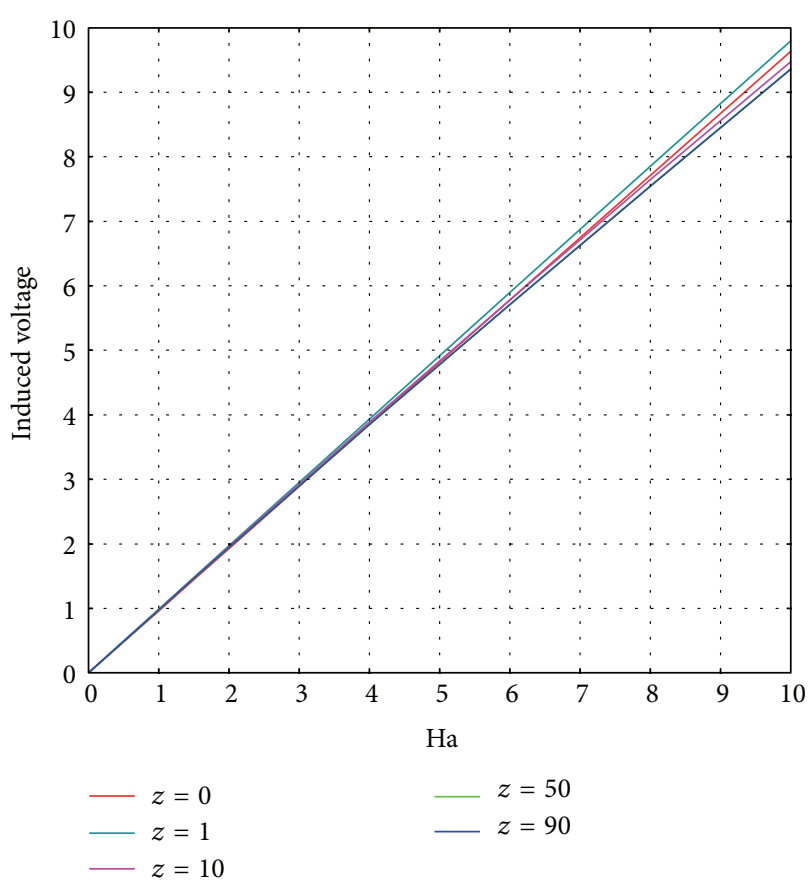

FIGURE 17: Effect of steady entrance length on induced voltage with Hartmann number Ha.

region thereby decreasing trend of velocity is observed in the centre.

Magnetically induced blood flow voltages are of greater importance in ECG signals. The effect of entrance length in the case of steady flow on induced voltage with Hartmann number $\mathrm{Ha}$ is shown in Figure 17. The induced voltage is found to be increased linearly with Hartmann number Ha. We observed that in both the cases induced voltage does not depend on the entrance length. We may demonstrate that the mean electric field is constant throughout the vessel (along longitudinal direction) as compared to mean velocity. This is the possible reason for induced voltage of independency on entrance length. Moreover, the results stated above are valid only for low Hartmann number $\mathrm{Ha}$ within the range of physiological systems, while the induced voltage has insignificant (however it may small) change on the axial position for higher values of Ha. It is also observed that the induced voltage has an oscillating effect with time.

\section{Conclusions}

The determination of steady and unsteady entrance length as well as the blood flow characteristics in the presence of magnetic field using computational fluid dynamics approach has been the main concern in this investigation. From our fully numerical solutions the main findings are summarized as follows.

(1) The steady entrance length is found to be increased with Reynolds number $\mathrm{Re}$ and decreased with the increase of Hartmann number Ha. The steady entrance length in terms of magnetic field strength as 
well as the Reynolds number Re can be approximated theoretically as presented in (22).

(2) The sinusoidal variation with time in cycle is observed for unsteady entrance length. The amplitude of the unsteady entrance length is greater for lower value of the Womersley parameter $\alpha$ and is minimum for larger values. The unsteady entrance length is also decreased with Hartmann number Ha. The phase difference is observed in unsteady entrance length for the presence of both the parameters $\alpha$ and Ha.

(3) During pulsatile blood flow, the reversal flow can be strongly suppressed by applying strong magnetic field. The Womersley parameter has reducing effect on velocity in the core region and an enhancing effect in the boundary layer during its peak flow, while the trend is reversed in the case of minimal flow rate.

(4) The interaction between induced currents and applied magnetic field causes reduction in flow velocity and thereby increases blood pressure in order to retain constant flow rate. The induced magnetic field forms two lobes on each side of the main current line and the induced currents recirculates inside the vessel, due to the consideration of nonconducting vessel walls. Thus the effect of induced magnetic field should not be overcome.

(5) The induced voltage is also varying sinusoidally with time and proportional to the applied magnetic field only. It is worth mentioning here that the induced voltage does not depend on the flow development.

Therefore, the study presented here is bearing important physiological phenomena and provides a lot of information to the scientist/researchers/experimentalists who are interested in cardiovascular flow simulation particularly in the patient-specific models. We may also conclude that finding analytical solutions with the interaction of electromagnetic field in unsteady case is not possible and thus the numerical simulation is the only way of its solution.

\section{Conflict of Interests}

The author declares that there is no conflict of interests regarding the publication of this paper.

\section{Acknowledgments}

The author is grateful to the esteemed reviewers for their suggestive and nice comments for which the present paper is revised. The author is thankful to the Department of Science \& Technology, Govternment of India for awarding him BOYSCAST fellowship (Reference no. SR/BY/M-01/09) to work at the University of Paris XII, France. The author would like to thank Professor C. Oddou, Universtiy of Paris XII, France, and Professor Philippe Dantan, University of Paris 7, France, for their valuable discussions.

\section{References}

[1] J. A. Shercliff, "The flow of conducting fluids in circular pipes under transverse magnetic fields," Journal of Fluid Mechanics, vol. 1, pp. 644-666, 1956.

[2] J. A. Shercliff, "Entry of conducting and non-conducting fluids in pipes," Mathematical Proceedings of the Cambridge Philosophical Society, vol. 52, pp. 573-583, 1956.

[3] H. B. Atabek and C. C. Chang, "Oscillatory flow near the entry of a circular tube," Zeitschrift für Angewandte Mathematik und Physik, vol. 12, no. 3, pp. 185-201, 1961.

[4] M. Van Dyke, "Entry flow in a channel," Journal of Fluid Mechanics, pp. 813-823, 1970.

[5] J. H. Gerrard and M. D. Hughes, "The flow due to an oscillating piston in a cylidrical tube: a comparison between experiment and a simple entrance flow theory," Journal of Fluid Mechanics, vol. 50, pp. 97-106, 1971.

[6] E. Kassianides and J. H. Gerrard, "The calculation of entrance length in physiological flow," Medical and Biological Engineering, vol. 13, no. 4, pp. 558-560, 1975.

[7] J. R. Womersley, "Method for the calculation of velocity, rate of flow and viscous drag in arteries when the pressure gradient is known., The Journal of Physiology, vol. 127, no. 3, pp. 553-563, 1955.

[8] J. R. Womersley, "Velocity profiles of oscillating arterial flow, with some calculations of viscous drag and the Reynolds number," The Journal of Physiology, vol. 128, pp. 629-640, 1955.

[9] X. He and D. N. Ku, "Unsteady entrance flow development in a straight tube," Journal of Biomechanical Engineering, vol. 116, no. 3, pp. 355-360, 1994.

[10] R. R. Gold, "Magnetohydrodynamics pipe flow, part-I," Journal of Fluid Mechanics, vol. 13, pp. 505-512, 1962.

[11] V. A. Vardanyan, "Effect of a magnetic field on blood flow," Biofizika, vol. 18, no. 3, pp. 515-521, 1973.

[12] D. Abi-Abdallah, A. Drochon, V. Robin, and O. Fokapu, "Effects of static magnetic field exposure on blood flow," The European Physical Journal Applied Physics, vol. 45, no. 1, p. 11301, 2009.

[13] J. Hartmann, "Hg-dynamics-I: theory of the laminar flow of an electrically conductive liquid in a homogeneous magnetic field," Mathematisk-Fysiske Meddeleser, vol. 15, no. 6, pp. 1-28, 1937.

[14] J. Hartmann and F. Lazarus, "Hg-dynamics-II: experimental investigations onthe flow of mercury in a homogeneous magnetic field," Mathematisk-Fysiske Meddeleser, vol. 15, no. 7, pp. $1-45,1937$.

[15] D. Abi-Abdallah, A. Drochon, V. Robin, and O. Fokapu, "Pulsed magnetohydrodynamic blood flow in a rigid vessel under physiological pressure gradient," Computer Methods in Biomechanics and Biomedical Engineering, vol. 12, no. 4, pp. 445-458, 2009.

[16] Y. Kinouchi, H. Yamaguchi, and T. S. Tenforde, "Theoretical analysis of magnetic field interactions with aortic blood flow," Bioelectromagnetics, vol. 17, no. 1, pp. 21-32, 1996.

[17] R. R. Raymond, C. C. Anaira, and L. W. Richard, "Exposure to strong static magnetic field slows the growth of human cancer cells in vitro," Bioelectromagnetics, vol. 17, no. 5, pp. 358-363, 1996.

[18] M. Jekic, Y. Ding, R. Dzwonczyk, P. Burns, S. V. Raman, and O. P. Simonetti, "Magnetic field threshold for accurate electrocardiography in the MRI environment," Magnetic Resonance in Medicine, vol. 64, no. 6, pp. 1586-1591, 2010.

[19] D. Formica and S. Silvestri, "Biological effects of exposure to magnetic resonance imaging: an overview," BioMedical Engineering Online, vol. 3, article 11, 2004. 
[20] T. S. Tenforde, "Interaction mechanisms and biological effects of static magnetic fields," Automedica, vol. 14, pp. 271-293, 1992.

[21] T. S. Tenforde, "Magnetically induced electric fields and currents in the circulatory system," Progress in Biophysics and Molecular Biology, vol. 87, no. 2-3, pp. 279-288, 2005.

[22] T. S. Tenforde, C. T. Gaffey, B. R. Moyer, and T. F. Budinger, "Cardiovascular alterations in Macaca monkeys exposed to stationary magnetic fields: experimental observations and theoretical analysis," Bioelectromagnetics, vol. 4, no. 1, pp. 1-9, 1983.

[23] P. Jehenson, D. Duboc, T. Lavergne et al., "Change in human cardiac rhythm induced by a 2-T static magnetic field," Radiology, vol. 166, no. 1, pp. 227-230, 1988.

[24] D. W. Chakeres, A. Kangarlu, H. Boudoulas, and D. C. Young, "Effect of static magnetic field exposure of up to 8 Tesla on sequential human vital sign measurements," Journal of Magnetic Resonance Imaging, vol. 18, no. 3, pp. 346-352, 2003.

[25] E. A. Bullard, "discussion on magnetohydrodynamics," Proceedings of the Royal Society A, vol. 233, pp. 289-296, 1955.

[26] J. Fabri and R. Siestrunck, "Contribution a la theorie aerodynamique du debitmetre electromagnetique," Bulletin de l'Association Technique Maritime et Aéronautique, vol. 60, pp. 333-350, 1960.

[27] F. Issacci, N. M. Ghoniem, and I. Catton, "Magnetohydrodynamic flow in a curved pipe," Physics of Fluids, vol. 31, pp. 65-71, 1988.

[28] R. Joulie, Mecanique des fluides Appliquee, Ellipses, Paris, France, 1998.

[29] R. Comolet, Mecanique experimentale des fluides, Tome-II, Masson, Paris, France, 1976.

[30] W. S. Janna, "Internal incompressible viscous flow," in Handbook of Fluid Dynamics, R. W. Johnson, Ed., CRC Press, Boca Raton, Fla, USA, 1998. 

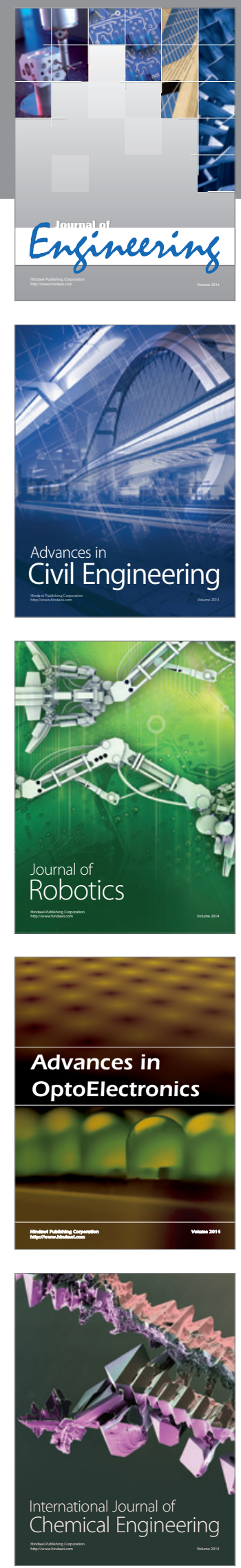

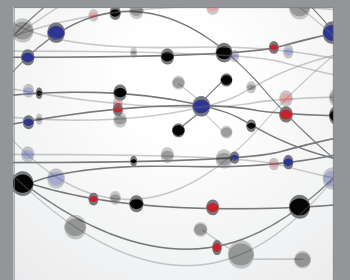

The Scientific World Journal
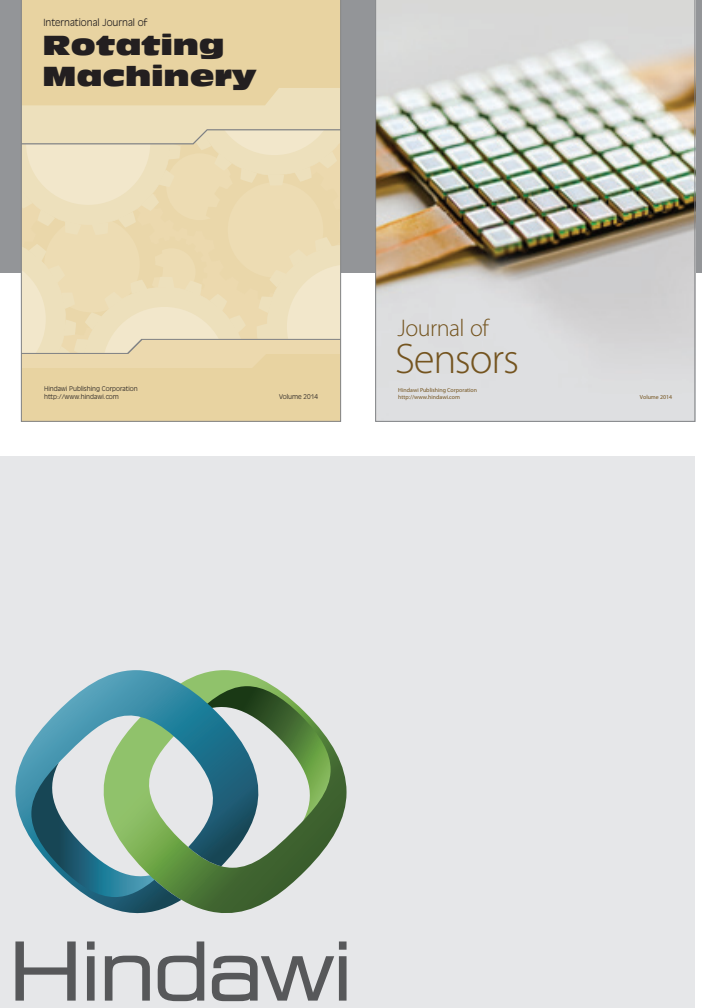

Submit your manuscripts at http://www.hindawi.com
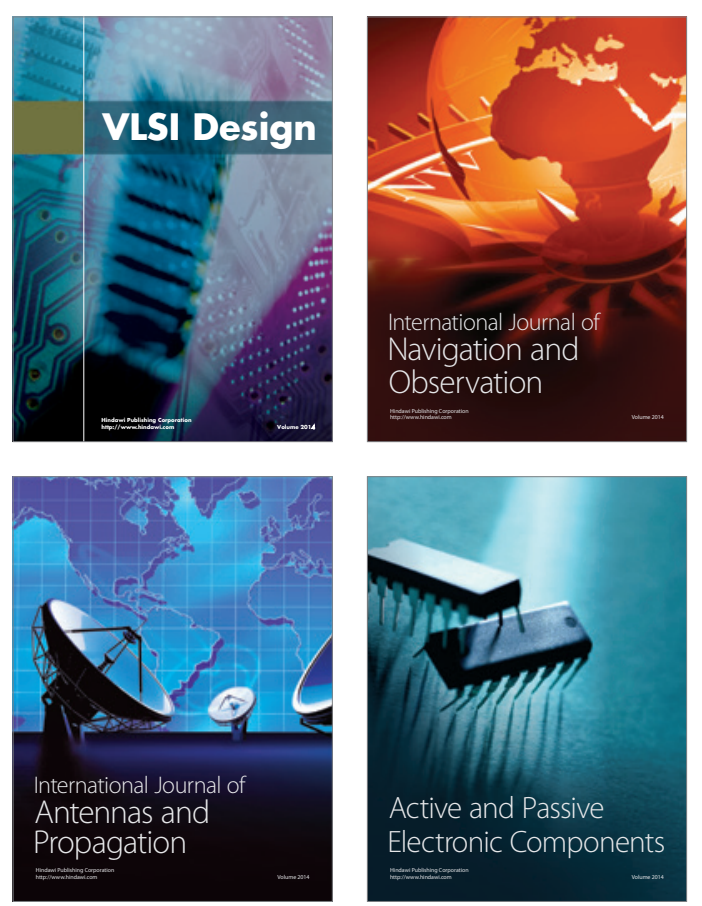
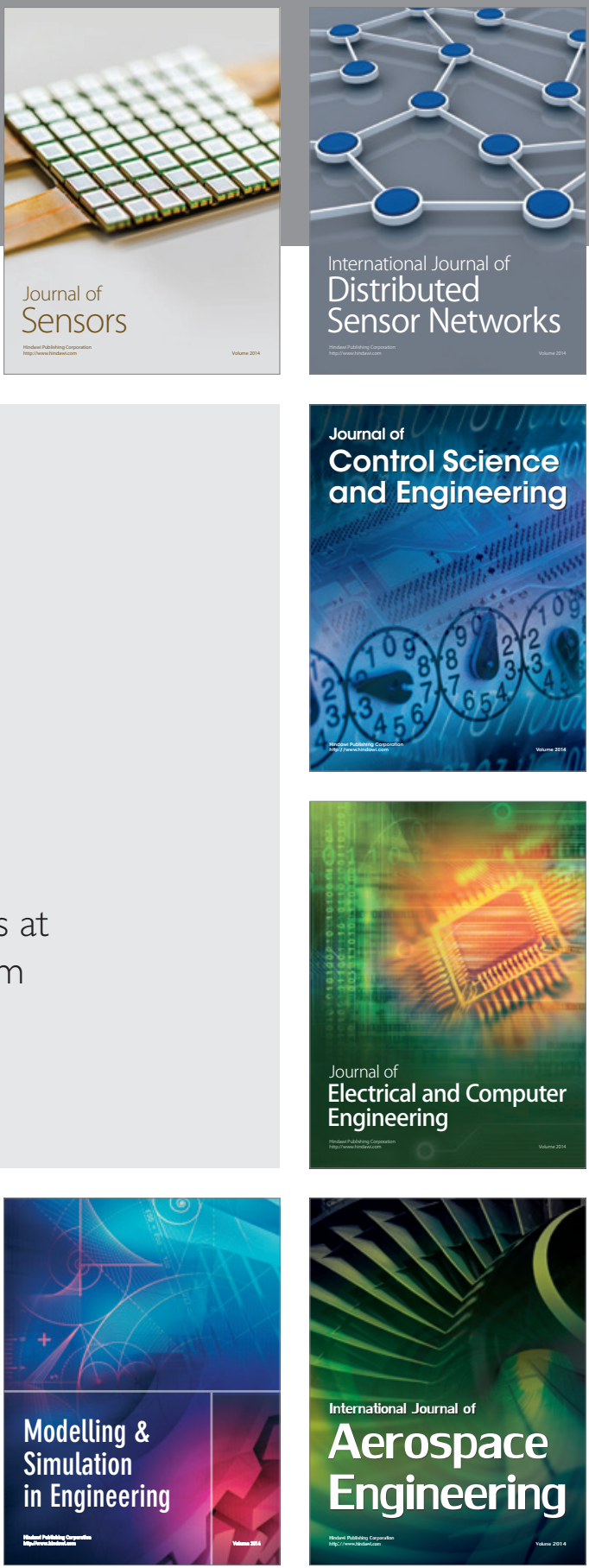

Journal of

Control Science

and Engineering
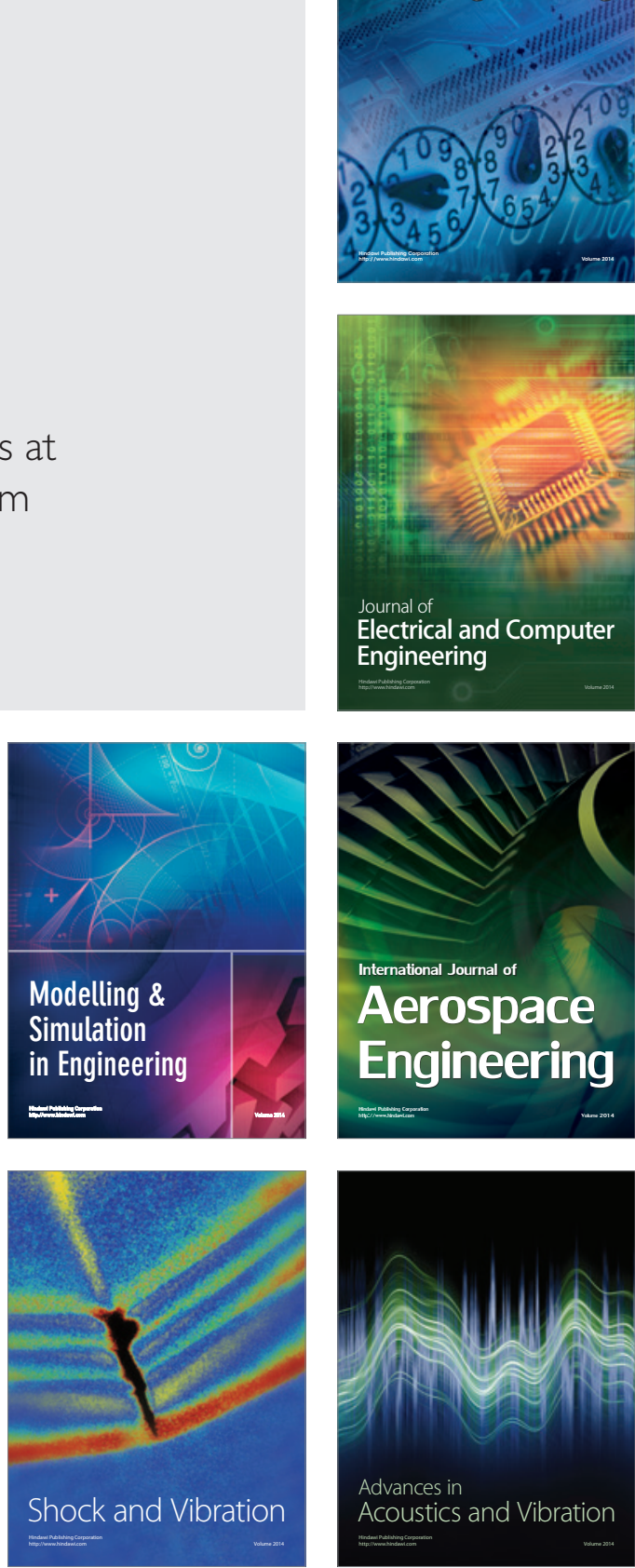